\title{
Antibiotic Translocation and Membrane Barrier: New Insights to Combat Bacterial Resistance Jean-Marie Pagès UMR_MD1, TMCD2, Aix-Marseille Univ, IRBA, Marseille, France ${ }^{\dagger}$
}

\author{
Jean-Marie Pagès \\ UMR_MD1, TMCD2, Aix-Marseille University, IRBA, 13005 Marseille, France; jean-marie.pages@univ-amu.fr \\ † Presented at the 1st Molecules Medicinal Chemistry Symposium, Barcelona, Spain, 8 September 2017. \\ Published: 16 October 2017
}

\section{Introduction}

The increasing prevalence of antibacterial resistance is a worrying health concern. A challenge in antibacterial research is to better understand membrane permeation of antibiotics in infective bacteria: passing the membrane barrier to reach the threshold of active concentration inside the bacterium is a pivotal step for all antibiotics. This is particularly acute for Gram-negative bacteria that have two membranes, the outer and the inner membranes that strongly limit the transport and the intracellular accumulation of antibiotics. A key point is to determine the real concentrations of antibiotics inside bacterial cells to determine the parameters modulating this internal accumulation.

\section{Methods}

Recently, new concepts, RTC2T and SICAR (Masi et al., Nat. Microbiol. 2, 17001 (2017) have been proposed to evaluate the relationship between membrane permeability and antibiotic accumulation. A spectrofluorimetric methodology has been developed to detect fluoroquinolones in bacterial population and inside individual Gram-negative bacterial cells. The antibiotic accumulation was studied in cells expressing various levels of efflux pumps.

\section{Results and Discussion}

The assays allow the determination of the intracellular concentration of the fluoroquinolones to study the relationships between the level of efflux activity and the antibiotic accumulation, and finally to evaluate the impact of fluoroquinolone structures in this process. This clearly validates the recently proposed "Structure Intracellular Concentration Activity Relationship" (SICAR) concept.

\section{Conclusions}

The combination of these studies that include drug imaging studies, evaluation of antibacterial activity and determination of membrane permeability, represents a promising research strategy. This strongly stimulates the molecular understanding of resistance mechanisms and the development of a future rational antibacterial chemotherapy.

Acknowledgments: The research leading to the results presented here was conducted as part of the translocation consortium (www.translocation.eu) and has received support from the Innovative Medicines Initiative joint Undertaking under Grant Agreement $n^{\circ} 115525$, resources which are composed of financial contribution from the European Union's seventh framework program (FP/2007-2013) and EFPIA companies in kind contributions. This work was also supported by Aix-Marseille Univ. and Service de Santé des Armées, and by Soleil program (projects \# 20130949, 20140047, 20141262, 20150318). 
Conflicts of Interest: The author declares no conflicts of interest.

\section{References}

1. Masi, M.; Réfregiers, M.; Pos, K.M.; Pagès, J.M. Mechanisms of envelope permeability and antibiotic influx/efflux in Gram negative bacteria. Nat. Microbiol. 2017, 2, 17001.

2. Zgurskaya, H.I.; López, C.A.; Gnanakaran, S. Permeability barrier of Gram-negative cell envelopes and approaches to bypass it. ACS Infect. Dis. 2015, 1, 512-522.

3. Cinquin, B.; Maigre, L.; Pinet, E.; Chevalier, J.; Stavenger, R.A.; Mills, S.; Réfrégiers, M.; Pagèsb, J. Microspectrometric insights on the uptake of antibiotics at the single bacterial cell level. Sci. Rep. 2015, 5, 17968.

4. Nikaido, H.; Pagès, J.M. Broad-specificity efflux pumps and their role in multidrug resistance of Gramnegative bacteria. FEMS Microbiol. Rev. 2012, 36, 340-363.

5. Kaščáková, S.; Maigre, L.; Chevalier, J.; Réfrégiers, M.; Pagès, J.M. Antibiotic transport in resistant bacteria: synchrotron UV fluorescence microscopy to determine antibiotic accumulation with single cell resolution. PLOS ONE 2012, 7, e38624.

(C) 2017 by the authors. Licensee MDPI, Basel, Switzerland. This article is an open access article distributed under the terms and conditions of the Creative Commons Attribution (CC BY) license (http://creativecommons.org/licenses/by/4.0/) 